\title{
Comparison of clinical characteristics, treatment, in-hospital and 12-month outcomes in patients after myocardial infarction with ejection fraction $<40 \%$ with or without atrial fibrillation
}

\author{
Przemysław Trzeciak ${ }^{1}$, Radosław Sierpiński²,3, Jacek Niedziela ${ }^{1}$, Wojciech Wojakowski", \\ Marek Gierlotka ${ }^{5}$, Mariusz Gąsior ${ }^{1}$, Zbigniew Kalarus ${ }^{6}$
}

\author{
${ }^{1} 3^{\text {rd }}$ Department of Cardiology, SMDZ in Zabrze, Medical University of Silesia, \\ Katowice, Silesian Center for Heart Diseases in Zabrze, Poland \\ ${ }^{2}$ Collegium Medicum, Kardynał Stefan Wyszynski Univeristy in Warsaw, Poland \\ ${ }^{3}$ National Institute of Cardiology in Warsaw, Poland \\ ${ }^{4}$ Department of Cardiology and Structural Heart Diseases, Medical University \\ of Silesia in Katowice, Poland \\ ${ }^{5}$ Department of Cardiology, University Hospital, Institute of Medical Sciences, \\ University of Opole, Poland \\ ${ }^{6}$ Clinical Department of Cardiology, Department of Cardiology, Congenital Heart \\ Disease and Electrotherapy, Silesian Center for Heart Diseases, Zabrze, Poland
}

Submitted: 27 May 2020

Accepted: 22 July 2020

Arch Med Sci

DOI: https://doi.org/10.5114/aoms.2020.100366

Copyright (c) 2020 Termedia \& Banach

\section{Abstract}

Introduction: The study aimed to compare the characteristics, treatment, and 12-month outcomes of patients after myocardial infarction (MI) and with left ventricular ejection fraction (LVEF) $<40 \%$, with or without atrial fibrillation (AF).

Material and methods: The analysis involved 10,222 surviving patients who were enrolled in the ongoing, prospective Polish Registry of Acute Coronary Syndromes (PL-ACS). The major adverse cardiac events (MACE) involved death, non-fatal MI, rehospitalization due to heart failure (HF), and stroke within 12 months after MI.

Results: Patients with AF were older (73.7 [13.1] vs. 68.3 [16.4], $p<0.001$ ) and had a greater prevalence of previous MI (37.3\% vs. $32.4 \%, p=0.0007)$, percutaneous coronary intervention (PCI) $(27.5 \%$ vs. $23.6 \%, p=0.002)$, and HF hospitalization $(34.6 \%$ vs. $19.7 \%, p<0.001)$. In patients with $\mathrm{AF}$, coronary angiography $(87.3 \%$ vs. $92.5 \%, p<0.001)$ and $\mathrm{PCl}(76.8 \%$ vs. $82.7 \%$, $p<0.001)$ were performed less frequently. Patients with AF had a higher rate of all-cause mortality ( $26.8 \%$ vs. $17.4 \%, p<0.001)$, HF hospitalization $(35.0 \%$ vs. $25.3 \%, p<0.001)$, stroke ( $5.5 \%$ vs. $2.1 \%, p<0.001)$ and MACE $(56.2 \%$ vs. $42.8 \%, p<0.001)$. In the multivariate analysis, AF was revealed to be associated with a higher all-cause mortality (odds ratio 1.20, 95\% confidence interval 1.03-1.40, $p=0.018$ ).

Conclusions: Patients after MI with LVEF $<40 \%$ and with AF, compared to those without $A F$, had worse clinical characteristics, were less frequently subjected to coronary angiography and $\mathrm{PCl}$ during hospitalization, and had significantly less favorable 12-month outcomes.

Key words: myocardial infarction, atrial fibrillation, long-term outcomes, left ventricular ejection fraction.
Corresponding author:

Przemysław Trzeciak MD, PhD $3^{\text {rd }}$ Department of Cardiology SMDZ in Zabrze

Medical University of Silesia Katowice Silesian Center for Heart Diseases in Zabrze Poland

E-mail: przemyslaw.t@wp.pl 


\section{Introduction}

One of the most important prognostic factors in patients after myocardial infarction (MI) is reduced left ventricular ejection fraction (LVEF). In the last three decades, LVEF has been used as the main diagnostic and prognostic parameter in the management of patients after $\mathrm{Ml}$ and heart failure (HF) [1-3]. Heart failure has been classified in the 2016 guidelines of the European Society of Cardiology (ESC) as HF with preserved ejection fraction (EF) (HFpEF, EF $\geq 50 \%$ ), HF with mid-range EF (HFmrEF, EF 40-49\%), and HF with reduced EF (HFrEF, EF < 40\%) [3].

One of the factors that seems to affect the long-term prognosis in patients with reduced LVEF is atrial fibrillation (AF) $[4,5]$. In patients with ST-segment elevation myocardial infarction (STEMI), the frequency of AF reaches up to $21 \%$ [6]. In a study of post-MI patients with LVEF $\leq 40 \%$, the 12-month incidence of AF was 32\% [7]. In the OPTIMAAL (Optima Trial in Myocardial Infarction with the Angiotensin II Antagonist Losartan) trial, patients after MI with signs of left ventricular dysfunction and concomitant AF had an increased risk of death and stroke during the follow-up period [8]. Atrial fibrillation is the most common arrhythmia in HF. It increases the risk of thromboembolic complications, particularly the risk of stroke. It may worsen cardiac function [3]. Therefore, patients with AF require special management and treatment. Notwithstanding, most of the papers focus mainly on the role of reduced LVEF on the long-term prognosis in patients after $\mathrm{MI}$, and they often pass over concomitant AF and its influence on long-term results in this group of patients $[2,9,10]$.

Due to data limitation, the present study aimed to compare the clinical characteristics, treatment, and 12 -month outcomes of patients after MI with $E F<40 \%$ and with or without $A F$, who survived index hospitalization and were enrolled in the Polish Registry of Acute Coronary Syndromes (PL-ACS).

\section{Material and methods}

\section{Registry design}

We used the data from the PL-ACS. The registry was established in 2003 and gathers detailed data on in-hospital management, the treatment, and the results of patients with acute coronary syndrome (ACS). The registry was a joint initiative of the Silesian Center for Heart Diseases in Zabrze and the Polish Ministry of Health. The design, methods, and logistic aspects of the PL-ACS registry have been described previously [11].

According to the protocol, all admitted patients with suspected ACS were screened for eligibility to enter the registry, though they were not enrolled until ACS had been confirmed. Patient data were collected by skilled physicians and entered directly using a dedicated web-based form.

\section{Data collection}

The follow-up data regarding the rates of allcause mortality, recurrent hospitalization, and stroke were available for a limited number of patients. The information was obtained from the Silesian Cardiovascular Database (SILCARD) and the Acute Myocardial Infarction in Poland (AMI-PL) registry. The SILCARD database was based on the agreement between the Silesian Center for Heart Diseases and the Regional Department of National Health Fund in Katowice to conduct a comprehensive analysis of patients with cardiovascular diseases in the Silesian Province [12]. The AMI-PL gathers administrative data from all MI hospitalizations recorded by the national healthcare provider. The detailed design and logistic aspects of SILCARD and AMI-PL databases have been presented previously [13].

\section{Endpoints and definitions}

Major adverse cardiovascular events (MACEs) involved death, non-fatal MI, rehospitalization due to $\mathrm{HF}$, and stroke within the 12-month follow-up. Death was considered as all-cause death. Non-fatal MI was defined as an ischemic event that met the ESC/ American College of Cardiology criteria for MI [14]. Stroke was recognized as an acute neurological deficit lasting $>24$ hours and affecting the possibility to perform daily activities or resulting in death. The analysis included only the patients with a confirmed diagnosis of STEMI and non-ST elevation myocardial infarction (NSTEMI). STEMI was defined as the presence of: 1) a typical anginal pain and/ or ischemic symptoms at rest lasting more than 20 minutes; 2) ST-segment elevation consistent with myocardial infarction of $2 \mathrm{~mm}$ or higher in the adjacent chest leads and/or ST-segment elevation of $1 \mathrm{~mm}$ or higher in 2 or more standard leads, or a new left bundle branch block; and 3) positive markers for cardiac necrosis. NSTEMI was defined as: 1) the absence of ST-segment elevation as defined above and 2) positive markers for cardiac necrosis.

\section{Study objectives}

The study population involved patients after MI with $\mathrm{EF}<40 \%$ divided into the two groups: with or without previous or on-admission AF. The analysis included only those patients who survived index hospitalization. We compared the differences in clinical characteristics, treatment strategy, as well as in-hospital and 12-month outcomes, including the number of MACEs. Finally, we identified the independent predictors of death and MACE incidence. 


\section{Statistical analysis}

All continuous variables had a skewed distribution and were presented as medians and interquartile ranges $[\mathrm{IQR}]$. Categorical variables were presented as percentages. We used the $\chi^{2}$ and Mann-Whitney $U$ tests as appropriate to test for differences between the patients with and without AF. A $p$-value of $<0.05$ was considered significant. Kaplan-Maier cumulative survival curves were constructed and the difference in mortality and MACE in the 12-month follow-up was compared using the log-rank test. To identify the independent predictors of 12-month outcomes for the final group of 10,231 patients, a logistic regression analysis was performed.

Forty-six variables with potentially predictive significance were used in the multivariate analysis, including baseline characteristics, comorbidities, hospital treatment, in-hospital events, and the treatment administered at admission. The stepwise forward regression was performed and only variables with a $p$-value of less than 0.05 were included. Analyses were performed using the Statistica 13.3 software (StatSoft).

\section{Results}

From October 2003 to April 2019, a total of 766,616 consecutive patients with ACS were enrolled in the ongoing, prospective PL-ACS registry. The final analysis involved 10,222 surviving patients with $\mathrm{MI}$ whose EF had been assessed during index hospitalization and the 12-month follow-up data. The study population flowchart is presented in Figure 1. The baseline demographic and clinical characteristics of patients with AF compared to those without AF are listed in Table I. The patients with AF were older and had a greater prevalence of previous $\mathrm{MI}$, percutaneous coronary intervention $(\mathrm{PCI})$, coronary artery bypass graft surgery, and HF hospitalization. Pulmonary edema, cardiogenic shock, and NSTEMI on admission were more frequent in the AF patients. LVEF was also significantly lower in the AF group. The in-hospital data are presented in Table II. In patients with AF, coronary angiography and $\mathrm{PCl}$ were performed less frequently. Patients from this group were more often diagnosed with multivessel coronary artery disease $(p<0.001)$.

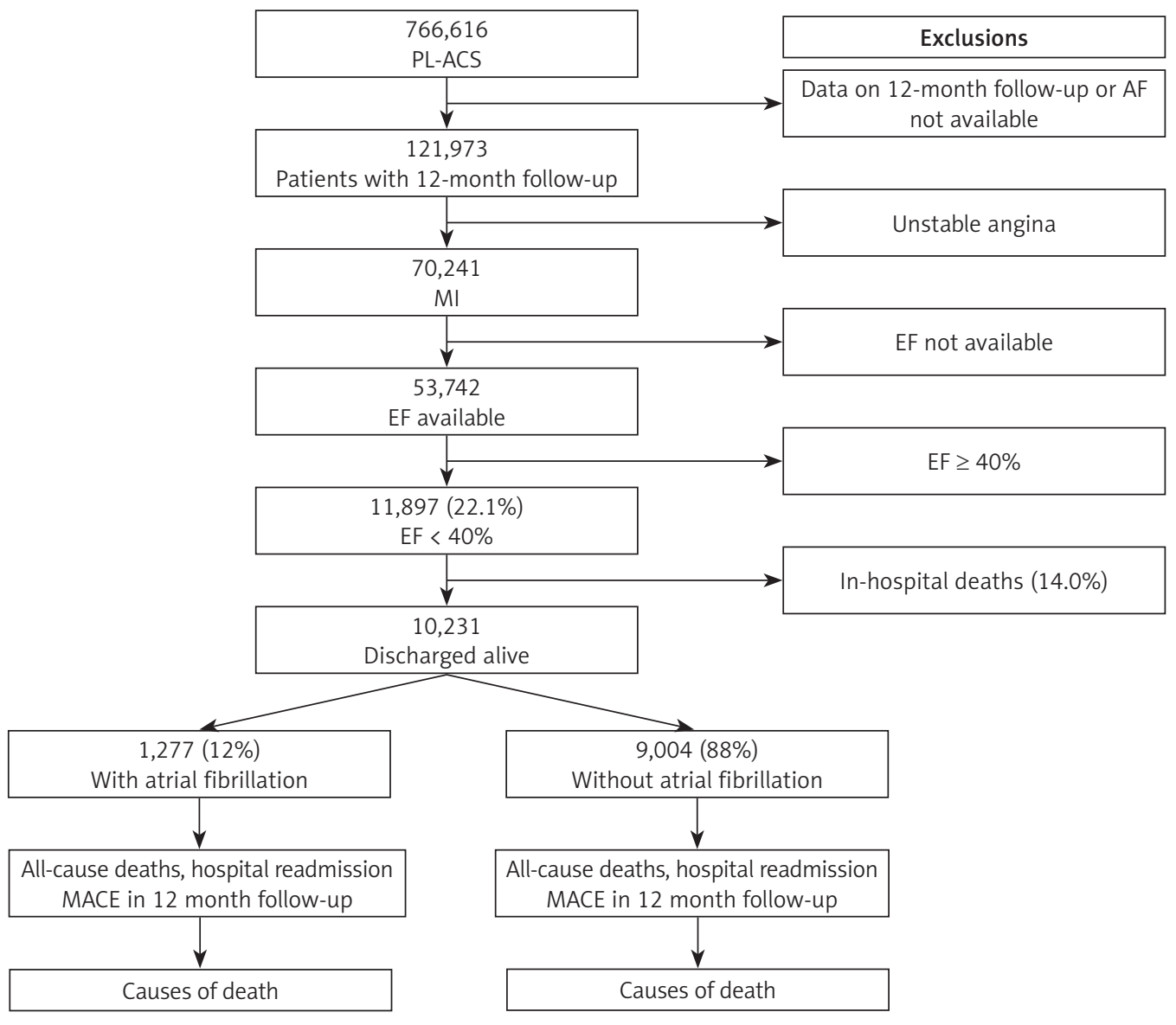

PL-ACS - Polish Registry of Acute Coronary Syndromes, AF - atrial fibrillation, MI- myocardial infarction, EF-ejection fraction, MACE - major adverse cardiovascular event.

Figure 1. Flowchart of the study 
Table I. Baseline demographic and clinical characteristics of the study groups

\begin{tabular}{|c|c|c|c|}
\hline Variables & $\begin{array}{c}\mathrm{AF} \\
(n=1,227)\end{array}$ & $\begin{array}{c}\text { Non-AF } \\
(n=8,995)\end{array}$ & $P$ \\
\hline Age [years], med [IQR] & $73.7[13.1]$ & $68.3[16.4]$ & $<0.001$ \\
\hline Males, $n(\%)$ & $802(65.4)$ & $6250(69.4)$ & 0.003 \\
\hline Previous MI, $n$ (\%) & $453(37.3)$ & $2907(32.4)$ & 0.0007 \\
\hline Previous $\mathrm{PCl}, n$ (\%) & $335(27.5)$ & $2116(23.6)$ & 0.002 \\
\hline Previous CABG, $n / n(\%)$ & $141 / 1217(11.6)$ & $802 / 8986(8.9)$ & 0.003 \\
\hline Previous stroke, $n(\%)$ & $105(10.6)$ & $397(5.7)$ & $<0.001$ \\
\hline Previous PM, $n$ (\%) & $17 / 248(6.9)$ & $19 / 933(2.0)$ & $0.0002 Y$ \\
\hline Previous ICD, $n(\%)$ & $25 / 248(10.1)$ & $28 / 933(3.0)$ & $<0.001 \mathrm{Y}$ \\
\hline Previous CRT-D, $n$ (\%) & $4 / 248(1.6)$ & $19 / 932(2.0)$ & $0.86 \mathrm{Y}$ \\
\hline Previous HF hospitalization, $n$ (\%) & $343(34.6)$ & $1367(19.7)$ & $<0.001$ \\
\hline $\mathrm{CHA}_{2} \mathrm{DS}_{2}$-VASc score, med [IQR] & $4[2]$ & $3[3]$ & $<0.0001$ \\
\hline $\mathrm{CHA}_{2} \mathrm{DS}_{2}$-VASc score (mean, SD) & $4.2(1.6)$ & $3.5(1.5)$ & $<0.0001$ \\
\hline Smoking, $n / n(\%)$ & $585 / 1179(49.6)$ & $5000 / 8828(56.6)$ & $<0.001$ \\
\hline Hypercholesterolemia, n (\%) & $619(51.5)$ & $4584(51.5)$ & 0.99 \\
\hline Diabetes mellitus, $n(\%)$ & $507(41.8)$ & $3153(35.6)$ & $<0.001$ \\
\hline Hypertension, $n$ (\%) & $967(79.1)$ & $6637(74.1)$ & 0.0002 \\
\hline Previous CKD, $n(\%)$ & $263(26.7)$ & $917(13.2)$ & $<0.001$ \\
\hline COPD, $n / n(\%)$ & $91 / / 984(9.2)$ & $433 / 6927(6.3)$ & 0.0004 \\
\hline Cancer, $n / n(\%)$ & $11 / 243(4.5)$ & $40 / 913(4.4)$ & $0.94 \mathrm{Y}$ \\
\hline \multicolumn{4}{|l|}{ Clinical presentation } \\
\hline OHCA, $n(\%)$ & $19(1.5)$ & $225(2.5)$ & $0.051 \mathrm{Y}$ \\
\hline NYHA I, $n(\%)$ & $251(27.3)$ & $2106(34.7)$ & $<0.001$ \\
\hline NYHA II, $n(\%)$ & $496(54.0)$ & $3148(51.8)$ & $<0.001$ \\
\hline NYHA III, $n(\%)$ & $154(16.8)$ & $740(12.2)$ & $<0.001$ \\
\hline NYHA IV, $n(\%)$ & $18(2.0)$ & $77(1.3)$ & $<0.001$ \\
\hline Pulmonary edema, $n(\%)$ & $116(9.5)$ & $636(7.1)$ & $<0.001$ \\
\hline Cardiogenic shock, $n(\%)$ & $50(4.1)$ & $389(4.3)$ & $<0.001$ \\
\hline STEMI, $n(\%)$ & $303(24.7)$ & $4083(45.4)$ & $<0.001$ \\
\hline SR (admission ECG), $n$ (\%) & $100(8.1)$ & $8560(95.5)$ & $<0.001$ \\
\hline AF (admission ECG), $n(\%)$ & $1115(90.9)$ & $405(4.5)$ & $<0.001$ \\
\hline LVEF, \% (mean, SD) & $28.7 \pm 6.7$ & $30.4 \pm 6.4$ & $<0.0001$ \\
\hline
\end{tabular}

$A F$ - atrial fibrillation, $I Q R$ - interquartile ranges, $M I$ - myocardial infarction, $P C I$ - percutaneous coronary intervention, CABG - coronary artery bypass grafting, $P M$ - pacemaker, $Y$ - Yates correction, ICD - implantable cardioverter defibrillator, CRT-D - cardiac resynchronization therapy defibrillator, HF - heart failure, CKD - chronic kidney disease, COPD - chronic obstructive pulmonary disease, OHCA - out-ofhospital cardiac arrest, NYHA - New York Heart Association, STEMI - ST-segment elevation myocardial infarction, SR - sinus rhythm, $L V E F$ - left ventricular ejection fraction, SD - standard deviation.

The 12-month outcomes are presented in Table III. Significantly higher mortality, HF hospitalization, as well as stroke and MACE rates were observed in patients with AF. Patients with
$\mathrm{AF}$ and a MACE during the follow-up period had a higher mean $\mathrm{CHA}_{2} \mathrm{DS}_{2}$-VASc score at admission compared to those without a MACE (Table IV). The Kaplan-Meier curves comparing the mortal- 
Comparison of clinical characteristics, treatment, in-hospital and 12-month outcomes in patients after myocardial infarction with ejection fraction $<40 \%$ with or without atrial fibrillation

Table II. In-hospital treatment procedures and pharmacotherapy at discharge

\begin{tabular}{|c|c|c|c|}
\hline Variables & AF & Non-AF & $P$ \\
\hline Coronary angiography, $n$ (\%) & $1062(87.3)$ & $8304(92.5 \%)$ & $<0.001$ \\
\hline \multicolumn{4}{|c|}{ Calculations in patients with coronary angiography } \\
\hline $\mathrm{PCl}, n / n(\%)$ & $816 / 1062(76.8)$ & $6867 / 8304(82.7)$ & $<0.001$ \\
\hline Radial artery approach, $n / n(\%)$ & $194 / 321(60.4)$ & $870 / 1326(65.0)$ & 0.13 \\
\hline Multivessel CAD, $n / n(\%)$ & $101 / 826(12.2)$ & $421 / 6827(6.2)$ & $<0.001$ \\
\hline GP IIb/IIla inhibitors, $n$ (\%) & $97(9.2)$ & $1374(16.6)$ & $<0.001$ \\
\hline TIMI 3 flow before $\mathrm{PCI}, n(\%)$ & $305(37.3)$ & $1644(24.0)$ & $<0.001$ \\
\hline TIMI 3 flow after PCI, $n$ (\%) & $723(89.6)$ & $6206(90.9)$ & 0.67 \\
\hline IABP support, $n(\%)$ & $14(1.3)$ & $237(2.9)$ & $0.005 \mathrm{Y}$ \\
\hline CABG during hospitalization, $n / n(\%)$ & $45 / 1059(4.2)$ & $358 / 8296(4.3)$ & $0.98 Y$ \\
\hline Pharmacotherapy at discharge & $n=1,226$ & $n=8,994$ & \\
\hline Aspirin, $n(\%)$ & $1121(91.4)$ & $8534(94.9 \%)$ & $<0.001$ \\
\hline $\mathrm{P} 2 \mathrm{Y} 12, n(\%)$ & 937 (76.4) & 7155 (79.5\%) & 0.01 \\
\hline \multicolumn{4}{|l|}{ Dual antiplatelet therapy: } \\
\hline 1-2 months, $n(\%)$ & $201(16.4)$ & $234(2.6)$ & $<0.001$ \\
\hline 3 months, $n(\%)$ & $59(4.8)$ & $54(0.6)$ & $<0.001$ \\
\hline 6 months, $n(\%)$ & $96(7.8)$ & $198(2.2)$ & $<0.001$ \\
\hline 12 months, $n(\%)$ & $838(68.4)$ & $7861(87.4)$ & $<0.001$ \\
\hline$>12$ months, $n(\%)$ & $21(2.6)$ & $648(7.2)$ & $<0.001$ \\
\hline Anticoagulants, $n(\%)$ & $476(38.8)$ & 976 (10.9) & $<0.001$ \\
\hline VKA, $n(\%)$ & $210(17.1)$ & $190(2.1)$ & $<0.001$ \\
\hline NOAC, $n(\%)$ & $87(7.1)$ & $35(0.4)$ & $<0.001$ \\
\hline $\mathrm{LMWH}, n(\%)$ & $227(18.5)$ & $787(8.7)$ & $<0.001$ \\
\hline$\beta$-blocker, $n(\%)$ & $1078(87.9)$ & $7859(87.4)$ & 0.64 \\
\hline ACEI, $n(\%)$ & $961(78.3)$ & $7328(81.4)$ & 0.01 \\
\hline ARBI, $n(\%)$ & $49(4.0)$ & $292(3.2)$ & $0.19 \mathrm{Y}$ \\
\hline ARNI, $n(\%)$ & $9(0.7)$ & $16(0.2)$ & $0.0003 \mathrm{Y}$ \\
\hline Diuretic, $n(\%)$ & $933(76.0)$ & $5300(58.9)$ & $<0.001$ \\
\hline Statin, $n(\%)$ & 1079 (87.9) & $8073(89.7)$ & 0.058 \\
\hline
\end{tabular}

$A F$ - atrial fibrillation, $P C I$ - percutaneous coronary intervention, CAD - coronary artery disease, GP - glycoprotein, TIMI - thrombolysis in myocardial infarction, IABP - intra-aortic balloon pump, $Y$ - Yates correction, CABG - coronary artery bypass grafting, VKA - vitamin $K$ antagonist, NOAC - new oral anticoagulant, LMWH - low molecular weight heparin, ACEI - angiotensin-converting enzyme inhibitor, $A R B$ - angiotensin receptor blocker, ARNI - angiotensin receptor-neprilysin inhibitor.

ity and MACE differences between the 2 groups during the 1-year follow-up showed a better prognosis for the patients without AF (Figures 2 and 3). In the multivariate analysis of the entire study population, AF was revealed to be one of the factors associated with a higher all-cause mortality $(\mathrm{OR}=1.20,95 \% \mathrm{Cl}: 1.03-1.40, p=0.018)$ and MACE incidence $(\mathrm{OR}=1.23,95 \% \mathrm{Cl}: 1.08-1.41, p=0.002)$ during the 12-month follow-up period (Tables $\mathrm{V}$ and $\mathrm{VI}$ ).

\section{Discussion}

Patients after MI with LVEF under $40 \%$ with $A F$, compared to those without AF, showed inferior in-hospital clinical characteristics, were less frequently subjected to coronary angiography 
Table III. Cardiac implantable electronic device implantation and events in 12-month follow-up

\begin{tabular}{|c|c|c|c|}
\hline Variables & $\begin{array}{c}\mathrm{AF} \\
n=1,227\end{array}$ & $\begin{array}{c}\text { Non-AF } \\
n=9,002\end{array}$ & $P$ \\
\hline \multicolumn{4}{|l|}{ CIED implantation } \\
\hline $\mathrm{PM}, n(\%)$ & $15(1.2)$ & $72(0.8)$ & $0.18 Y$ \\
\hline $\mathrm{ICD}, n(\%)$ & $87(7.1)$ & $573(6.4)$ & $<0.001$ \\
\hline CRT-D, $n(\%)$ & $18(1.5)$ & $126(1.4)$ & $0.69 \mathrm{Y}$ \\
\hline CRT-D or CRT-P, $n(\%)$ & $18(1.5)$ & $130(1.4)$ & $0.79 \mathrm{Y}$ \\
\hline \multicolumn{4}{|l|}{ Events } \\
\hline Hospital readmission (any), $n$ (\%) & $923(75.2)$ & $6651(73.9)$ & 0.32 \\
\hline Death during hospitalization, $n(\%)$ & $198(16.1)$ & $818(9.1)$ & $<0.001$ \\
\hline Death out of hospital, $n(\%)$ & $130(10.7)$ & $749(8.3)$ & 0.008 \\
\hline All-cause mortality, $n(\%)$ & $328(26.8)$ & $1567(17.4)$ & $<0.001$ \\
\hline MI, $n(\%)$ & $125(10.2)$ & $984(10.9)$ & 0.43 \\
\hline HF hospitalization, $n(\%)$ & $429(35.0)$ & $2276(25.3)$ & $<0.001$ \\
\hline Stroke, $n(\%)$ & $68(5.5)$ & $187(2.1)$ & $<0.001$ \\
\hline MACE, $n(\%)$ & $690(56.2)$ & $3856(42.8)$ & $<0.001$ \\
\hline
\end{tabular}

$A F$ - atrial fibrillation, CIED - cardiac implantable electronic device, PM - pacemaker, ICD - implantable cardioverter defibrillator, $C R T$-D - cardiac resynchronization therapy defibrillator, $Y$ - Yates correction, CRT-P-cardiac resynchronization therapy pacemaker, MI - myocardial infarction, HF - heart failure, MACE - major adverse cardiac events.

Table IV. Values of CHA2DS2-VASc score in atrial fibrillation patients with or without MACE incidence during the follow-up period

\begin{tabular}{|c|c|c|c|}
\hline Variables & $\begin{array}{c}\mathrm{CHA}_{2} \mathrm{DS}_{2}-\text { VASc score in patients } \\
\text { with MACE }\end{array}$ & $\begin{array}{c}\mathrm{CHA}_{2} \mathrm{DS}_{2}-\text { VASc score in patients } \\
\text { without MACE }\end{array}$ & $P$ \\
\hline MACE (mean, SD) & $4.3(1.5)$ & $4.0(1.6)$ & 0.007 \\
\hline MACE, med (IQR) & $4(2)$ & $4(2)$ & 0.01 \\
\hline Stroke (mean, SD) & $4.6(1.5)$ & $4.14(1.6)$ & 0.02 \\
\hline All-cause death (mean, SD) & $4.5(1.5)$ & $4.05(1.6)$ & $<0.0001$ \\
\hline
\end{tabular}

and $\mathrm{PCl}$ during hospitalization, and had worse 12-month outcomes.

Reduced LVEF in patients after $\mathrm{MI}$ is a predictor of poor prognosis [2]. The plurality of clinical characteristics and the long-term prognosis of patients after MI with LVEF < $40 \%$ is still unappreciated. The majority of randomized trials evaluating therapeutic or mechanical interventions have been restricted to patients with symptomatic congestive heart failure (CHF) and impaired left ventricular function [9, 15-17]. The number of papers assessing factors that affect the long-term prognosis in patients after $\mathrm{MI}$ with impaired left ventricular function is still insufficient. AF seems to be an underestimated factor. Patients with AF have more comorbidities and have a higher risk of complications [4, 18, 19].

The available reports assessing the influence of $\mathrm{AF}$ on the long-term outcomes in patients with
LVEF $<40 \%$ are restricted to those with HFrEF [20-23]. The published data come mainly from scanty HF registries. The HF Long-Term Registry of the ESC includes data collected in 21 countries from 12,440 patients. Over $40 \%$ of them were hospitalized with acute HF and nearly $60 \%$ were outpatients with chronic HF [24]. The Swedish Heart Failure Registry (SwedeHF) is a prospective, ongoing registry that records data at discharge from hospitals or after outpatient clinic visits [22]. The Korean Acute Heart Failure Registry (KorAHF) was a prospective registry that enrolled patients hospitalized with acute HF at tertiary university hospitals [23, 25, 26].

In patients with HFrEF, the prevalence of $\mathrm{AF}$ ranges from $<10 \%$ to $53 \%[22,27,28]$. The prognostic impact of $A F$, according to different types of HF, especially HFrEF, has not been fully evaluated. In a population of 14,964 patients included in 
Table V. Multivariate analysis. All-cause mortality in 12-month follow-up after discharge

\begin{tabular}{|c|c|c|c|}
\hline Factor & OR & $95 \% \mathrm{Cl}$ & $P$ \\
\hline Age, per 1 year increase & 1.041 & $1.035-1.047$ & $<0.001$ \\
\hline NYHA III-IV (vs. I-II) & 1.28 & $1.08-1.51$ & 0.004 \\
\hline Killip 3-4 (vs. 1-2) & 1.24 & $1.07-1.44$ & 0.005 \\
\hline EF, per 1 percent increase & 0.96 & $0.95-0.97$ & $<0.001$ \\
\hline$A F$ & 1.20 & $1.03-1.40$ & 0.018 \\
\hline Hypercholesterolemia & 0.84 & $0.76-0.94$ & 0.002 \\
\hline Diabetes mellitus & 1.30 & $1.17-1.45$ & $<0.001$ \\
\hline Previous CKD & 1.49 & $1.28-1.74$ & 0.005 \\
\hline Smoking & 1.16 & $1.04-1.31$ & 0.011 \\
\hline Radial approach (vs. other) & 0.65 & $0.49-0.86$ & 0.003 \\
\hline $\mathrm{ACEI}$ or $\mathrm{ARB}$ or $\mathrm{ARNI}$ at discharge & 0.75 & $0.65-0.86$ & $<0.001$ \\
\hline Beta-blocker at discharge & 0.79 & $0.67-0.92$ & 0.003 \\
\hline Diuretics at discharge & 1.18 & $1.05-1.32$ & 0.006 \\
\hline Cardiogenic shock during hospitalization & 1.93 & $1.36-2.74$ & $<0.001$ \\
\hline
\end{tabular}

$O R$ - odds ratio, Cl - confidence interval, NYHA - New York Heart Association, EF - ejection fraction, AF-atrial fibrillation, CKD - chronic kidney disease, ACEI - angiotensin-converting enzyme inhibitor, ARB - angiotensin receptor blocker, ARNI-angiotensin receptor-neprilysin inhibitor.

Table VI. Multivariate analysis. Major adverse cardiac events in 12-month follow-up after discharge

\begin{tabular}{|lccc|}
\hline Factor & OR & $95 \%$ Cl & $P$ \\
\hline Age, per 1 year increase & 1.018 & $1.014-1.022$ & 0.001 \\
\hline NYHA III-IV (vs. I-II) & 1.29 & $1.11-1.49$ & 0.001 \\
\hline Killip 3-4 (vs. 1-2) & 1.20 & $1.05-1.36$ & $<.007$ \\
\hline EF, per 1 percent increase & 0.95 & $0.94-0.96$ & 0.001 \\
\hline STEMI (vs. NSTEMI) & 0.89 & $0.81-0.98$ & 0.002 \\
\hline AF & 1.23 & $1.08-1.41$ & $<0.001$ \\
\hline Diabetes mellitus & 1.29 & $1.17-1.45$ & 0.001 \\
\hline Radial approach (vs. other) & 0.71 & $0.57-0.88$ & 0.001 \\
\hline ACEI or ARB or ARNI at discharge & 0.83 & $0.74-0.92$ & $<0.001$ \\
\hline Diuretics at discharge & 1.20 & $1.10-1.31$ & 0.003 \\
\hline Cardiogenic shock during hospitalization & 1.64 & $0.67-0.92$ & $<$ \\
\hline
\end{tabular}

MACE - major adverse cardiac events, OR - odds ratio, Cl - confidence interval, NYHA - New York Heart Association, EF - ejection fraction, STEMI - ST-segment elevation myocardial infarction, NSTEMI - non-ST-segment elevation myocardial infarction, AF - atrial fibrillation, $A C E I$ - angiotensin-converting enzyme inhibitor, ARB - angiotensin receptor blocker, ARNI-angiotensin receptor-neprilysin inhibitor.

the ESC-HF long-term registry, Zafrir et al. found that all-cause death and HF hospitalization rates were higher in patients with AF compared to those with sinus rhythm (SR) in each of the three EF groups [20]. In the KorAHF registry, AF was found to be associated with increased mortality only in patients with HFpEF, but not in those with HFrEF and HFmrEF [23]. In a cohort of 41,446 patients enrolled in the SwedeHF registry, patients with AF compared to those with SR in each of the three EF groups had an increased risk of death, HF hospitalization, and stroke [22]. Similarly, in our analysis, patients with AF had a higher incidence of all-cause mortality, HF hospitalization, stroke, and MACE during the 12-month follow-up period.

Unfavorable long-term outcomes of AF patients require an explanation. Patients with AF compared to those with $\mathrm{SR}$ are older, have a higher rate of previous HF hospitalizations and inferior clinical characteristics [20, 22]. In the ESC-HF longterm registry, after a multivariable adjustment, AF was associated in each of the three HF subtypes with older age, reduced functional capacity, previous HF hospitalizations, higher heart rates, 


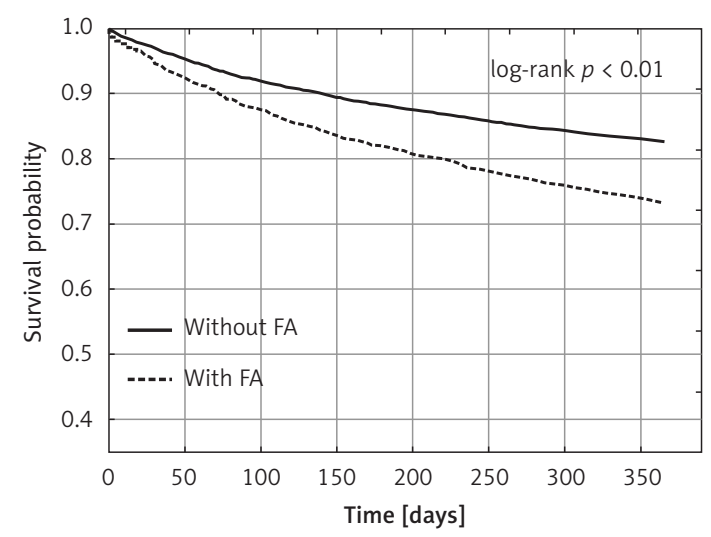

Figure 2. All-cause mortality in 12-month follow-up in patients without and with atrial fibrillation. KaplanMeier curves

and more significant signs of congestion [20]. In the SwedeHF registry, patients with AF compared to those with SR were older and were characterized by a longer duration and more severe HF, lower creatinine clearance, and more frequent hypertension, TIA, or stroke. These patterns were similar in all three HF groups [22]. In our study, patients with LVEF $<40 \%$ and concomitant AF were older, had a higher rate of previous $\mathrm{MI}$, revascularization procedures, CKD, and HF hospitalizations. Due to inclusive criteria used by our registry, as well as the cited ones, the study groups were heterogeneous. Therefore, the comparisons of patients with and without AF might be methodically questionable. In spite of that, a common feature of all the registries is the older age of patients with $\mathrm{AF}$ and the differences in basal characteristics of patients with and without AF. The long-term results are not analogous. Both in the HF Long-Term Registry and the SwedeHF Registry, patients with AF had higher mortality and worse long-term outcomes. In the KorAHF Registry, patients differed in the stroke rate, without a significant difference in the mortality rate in the long-term follow-up period.

Surprisingly, less than $40 \%$ of patients with AF were prescribed anticoagulants at discharge. Moreover, only $17.1 \%$ were recommended to be treated with vitamin $\mathrm{K}$ antagonists (VKA), 7.1\% with new oral anticoagulants, and $18.5 \%$ with low molecular weight heparin. Our analysis involved patients enrolled in the Registry from 2003 . The advantages of the anticoagulant therapy were less evident at that time. Low molecular weight heparins were not infrequently prescribed as the bridge therapy in patients treated ultimately with VKA.

Our multivariate analysis revealed AF to be an independent predictor of death and MACE incidence after discharge. However, whether AF is associated with less favorable outcomes in HFrEF patients remains controversial [22]. In the ESC-HF long-term registry, AF in patients with $\mathrm{HFpEF}$ and HFmrEF

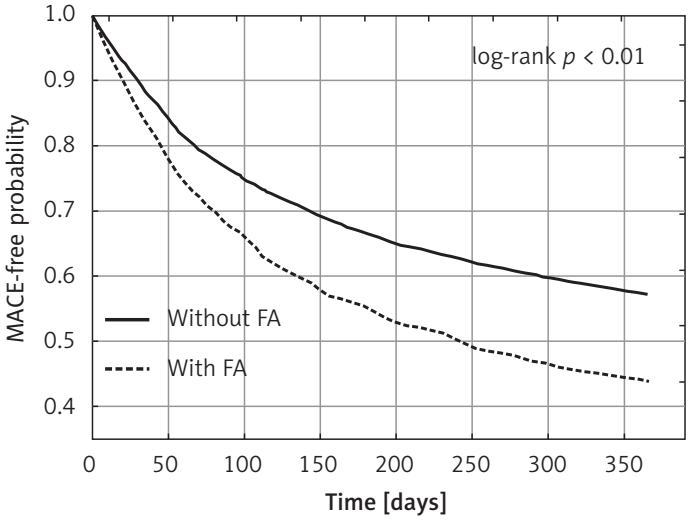

Figure 3. Major adverse cardiovascular event incidence in 12-month follow-up in patients without and with atrial fibrillation. Kaplan-Meier curves

was correlated with inferior cardiovascular outcomes, including death, though this was not true in the case of patients with HFrEF [20]. In the Framingham Heart Study, AF was associated with a similar risk of death in the incident HFpEF and HFrEF [29]. In the CHARM study, AF was associated with a relatively greater risk of the major outcomes in patients with a preserved $\mathrm{EF}$ than in patients with a low EF [30]. In the SwedeHF registry, the association of AF with outcomes and hazard ratios ranging from 1.11 to 1.29 for the three EF groups was similar [22]. A multivariate analysis showed protective effects of hypercholesterolemia and $\mathrm{PCl}$ performed in myocardial infarction from the radial artery access, and a better prognosis of patients with STEMI compared to those with NSTEMI. Patients undergoing surgery using the radial artery access are exposed to fewer hemorrhagic complications, which is particularly important in patients with AF treated with chronic anticoagulation. Patients with STEMI infarction are generally younger, have fewer associated diseases, and the identification of the vessel responsible for the infarction is generally more unambiguous in their case.

The study has strengths and limitations. The analysis involved only surviving patients, excluding those who died during the index hospitalization. Some other limitations are typical for registry-based cohort studies. Firstly, the treatment and in-hospital events may be underreported, probably due to incomplete records in the database. For example, LVEF was available for $76.5 \%$ of patients with MI. Secondly, as reporting to the registry was not obligatory, not all patients with $\mathrm{MI}$ in Poland were reported. A detailed follow-up was also available only for a limited number of patients. Thirdly, as our research is an observational study, some bias related to the lack of possibility of adjusting all variables might influence the final results. The causality of the results cannot be confirmed, as the study was retrospective. For these reasons, all results 
should be interpreted with caution. The subject still requires a further investigation, preferably in the form of a prospective study.

Our study also has some strengths. The PL-ACS Registry provides an opportunity to investigate, in a reliable manner, the treatment and outcomes of patients encountered in general practice. Among them, a longitudinal observation of a large number of patients, as well as detailed data on the medical history, treatment at discharge, and events within the follow-up period, can be highlighted. Moreover, the study showed the natural history of MI patients in real world settings. Finally, our research provided a hypothesis for further studies.

In conclusion, patients after MI with LVEF $<40 \%$ with $\mathrm{AF}$, compared to those without $\mathrm{AF}$, had inferior clinical characteristics, were less frequently subjected to coronary angiography and $\mathrm{PCl}$ during hospitalization, and - finally - had significantly less favorable 12-month outcomes, with higher rates of death and MACE.

\section{Acknowledgment}

The authors would like to express their gratitude to all the physicians and nurses who participated in the PL-ACS registry, the regional coordinators, and the employees of the National Health Fund of Poland for their logistic support.

The PL-ACS Registry is financed by the Ministry of Health.

\section{Conflict of interest}

The authors declare no conflict of interest.

\section{References}

1. Lauritsen J, Gustafsson F, Abdulla J. Characteristics and long-term prognosis of patients with heart failure and mid-range ejection fraction compared with reduced and preserved ejection fraction: a systematic review and meta-analysis. ESC Heart Fail 2018; 5: 685-94.

2. Ibánez B, James S, Agewall S, et al. ESC Guidelines for the management of acute myocardial infarction in patients presenting with ST-segment elevation. Rev Esp Cardiol 2017; 70: 1082.

3. Ponikowski P, Voors AA, Anker SD, et al. 2016 ESC Guidelines for the diagnosis and treatment of acute and chronic heart failure. Eur Heart J 2016; 37: 2129-200.

4. Batra G, Svennblad B, Held C, et al. All types of atrial fibrillation in the setting of myocardial infarction are associated with impaired outcome. Heart 2016; 102: 926-33.

5. Podolecki, T, Lenarczyk R, Kowalczyk J, et al. Significance of atrial fibrillation complicating ST-segment elevation myocardial infarction. Am J Cardiol 2017; 120: 517-21.

6. Schmitt J, Duray G, Gersh BJ, et al. Atrial fibrillation in acute myocardial infarction: a systematic review of the incidence, clinical features and prognostic implications. Eur Heart J 2009; 30: 1038-45.

7. Jons C, Jacobsen UG, Joergensen RM, et al. The incidence and prognostic significance of new-onset atrial fibrillation in patiens with acute myocardial infarction and left ventricular systolic dysfunction: a CARISMA substudy. Heart Rhythm 2011; 8: 342-8.

8. Lehto M, Snapinn S, Dickstein K, Swedberg K, Nieminen MS. Prognostic risk of atrial fibrillation in acute myocardial infraction complicated by left ventricular dysfunction: the OPTIMAAL experience. Eur Heart J 2005; 26: 350-6.

9. Moss AJ, Zareba W, Hall WJ, et al. Prophylactic implantation of a defibrillator in patients with myocardial infarction and reduced ejection fraction. N Engl J Med 2002; 346: 877-83.

10. Wita K, Kułach A, Wita M, et al. Managed Care after Acute Myocardial Infarction (KOS-zawał) reduces major adverse cardiovascular events by $45 \%$ in 3-month follow-up single-center results of Poland's National Health Fund program of comprehensive post-myocardial infarction care. Arch Med Sci 2019; 16: 551-8.

11. Poloński L, Gąsior M, Gierlotka M, et al. Polish Registry of Acute Coronary Syndromes (PL-ACS). Characteristics, treatments and outcomes of patients with acute coronary syndromes in Poland. Kardiol Pol 2007; 65: 861-72.

12. Gąsior M, Pres D, Wojakowski W, et al. Causes of hospitalization and prognosis in patients with cardiovascular diseases. Secular trends in the years 2006-2014 according to the SILesian CARDiovascular (SILCARD) database. Pol Arch Med Wewn 2016; 126: 754-62.

13. Gierlotka M, Zdrojewski T, Wojtyniak B, et al. Incidence, treatment, in-hospital mortality and one-year outcomes of acute myocardial infarction in Poland in 2009-2012 nationwide AMI-PL database. Kardiol Pol 2015; 73: 142-58.

14. Thygesen K, Alpert JS, White HD, Joint ESC/ACCF/AHA/ WHF Task Force for the redefinition of myocardial infarction. Universal definition of myocardial infarction. Joint ESC/ACCF/AHA/WHF task force for the redefinition of myocardial infarction. Eur Heart J 2007; 28: 2525-38.

15. Bardy GH, Lee KL, Mark DB, et al. Amiodarone or an implantable cardioverter-defibrillator for congestive heart failure. N Engl J Med 2005; 352: 225-37.

16. Goldenberg I, Moss AJ, McNitt S, et al. Time dependence of defibrillator benefit after coronary revascularization in the Multicenter Automatic Defibrillator Implantation Trial (MADIT)-II. J Am Coll Cardiol 2006; 47: 1811-7.

17. Zareba W, Klein H, Cygankiewicz I, et al. Effectiveness of cardiac resynchronization therapy by QRS morphology in the Multicenter Automatic Defibrillator Implantation Trial-Cardiac Resynchronization Therapy (MADIT-CRT). Circulation 2011; 123: 1061-72.

18. Piotrowski R, Zaborska B, Pilichowska-Paszkiet E, SikoraFrąc M, Baran J, Kułakowski P. RIVAroxaban TWICE daily for lysis of thrombus in the left atrial appendage in patients with non-valvular atrial fibrillation: the RIVATWICE study. Arch Med Sci 2019; 16: 289-96.

19. Kotlęga D, Gołąb-Janowska M, Meller A, et al. Beneficial effects of pre-stroke statins use in cardioembolic stroke patients with atrial fibrillation: a hospital-based retrospective analysis. Arch Med Sci 2019; 15: 385-92.

20. Zafrir B, Lund LH, Laroche C, et al. Prognostic implications of atrial fibrillation in heart failure with reduced, mid-range, and preserved ejection fraction: a report from 14964 patients in the European Society of Cardiology Heart Failure Long-Term Registry. Eur Heart J 2018; 39: 4277-84.

21. Xu HX, Zhu YM, Hua Y, Huang YH, Lu Q. Association between atrial fibrillation and heart failure with different ejection fraction categories and its influence on outcomes. Acta Cardiol 2020; 75: 423-32. 
22. Sartipy U, Dahlström U, Fu M, Lund LH. Atrial fibrillation in heart failure with preserved, mid-range, and reduced ejection fraction. JACC Heart Fail 2017; 5: 565-74.

23. Son MK, Park JJ, Lim NK, Kim WH, Choi DJ. Impact of atrial fibrillation in patients with heart failure and reduced, mid-range or preserved ejection fraction. Heart 2020; doi: 10.1136/heartjnl-2019-316219.

24. Crespo-Leiro MG, Anker SD, Maggioni AP, et al. European Society of Cardiology Heart Failure Long-Term Registry (ESC-HF-LT): 1-year follow-up outcomes and differences across regions. Eur J Heart Fail 2016; 18: 613-25.

25. Lee SE, Cho HJ, Lee HY, et al. A multicentre cohort study of acute heart failure syndromes in Korea: rationale, design, and interim observations of the Korean acute heart failure (KorAHF) registry. Eur J Heart Fail 2014; 16: 700-8.

26. Lee SE, Lee HY, Cho HJ, et al. Clinical characteristics and outcome of acute heart failure in Korea: results from the Korean acute heart failure registry (KorAHF). Korean Circ J 2017; 47: 341-53.

27. Stevenson WG, Stevenson LW, Middlekauff HR, et al. Improving survival for patients with atrial fibrillation and advanced heart failure. J Am Coll Cardiol 1996; 28 : 1458-63.

28. Maisel WH, Stevenson LW. Atrial fibrillation in heart failure: epidemiology, pathophysiology, and rationale for therapy. Am J Cardiol 2003; 91 (6A): 2D-8D.

29. Santhanakrishnan R, Wang N, Larson MG, et al. Atrial fibrillation begets heart failure and vice versa: temporal associations and differences in preserved versus reduced ejection fraction. Circulation 2016; 133: 484-92.

30. Olsson LG, Swedberg K, Ducharme A, et al. Atrial fibrillation and risk of clinical events in chronic heart failure with and without left ventricular systolic dysfunction: results from the Candesartan in Heart failure-Assessment of Reduction in Mortality and morbidity (CHARM) program. J Am Coll Cardiol 2006; 47: 1997-2004. 